\title{
Measurement of the Dynamics of the Decays $D_{s}^{+} \rightarrow \eta^{(\prime)} e^{+} \nu_{e}$
}

M. Ablikim, ${ }^{1}$ M. N. Achasov, ${ }^{10, d}$ S. Ahmed,${ }^{15}$ M. Albrecht,${ }^{4}$ M. Alekseev, ${ }^{55 a, 55 c}$ A. Amoroso, ${ }^{55 a, 55 c}$ F. F. An, ${ }^{1}$ Q. An,${ }^{52,42}$ J. Z. Bai, ${ }^{1}$ Y. Bai, ${ }^{41}$ O. Bakina, ${ }^{27}$ R. Baldini Ferroli, ${ }^{23 a}$ Y. Ban, ${ }^{35}$ K. Begzsuren, ${ }^{25}$ D. W. Bennett, ${ }^{22}$ J. V. Bennett, ${ }^{5}$ N. Berger, ${ }^{26}$ M. Bertani, ${ }^{23 a}$ D. Bettoni, ${ }^{24 a}$ F. Bianchi, ${ }^{55 a, 55 c}$ E. Boger, ${ }^{27, b}$ I. Boyko, ${ }^{27}$ R. A. Briere, ${ }^{5}$ H. Cai, ${ }^{57}$ X. Cai, ${ }^{1,42}$ A. Calcaterra, ${ }^{23 \mathrm{a}}$ G. F. Cao, ${ }^{1,46}$ S. A. Cetin, ${ }^{45 \mathrm{~b}}$ J. Chai,${ }^{55 \mathrm{c}}$ J. F. Chang, ${ }^{1,42}$ G. Chelkov, ${ }^{27, b, \mathrm{c}}$ G. Chen, ${ }^{1}$ H. S. Chen, ${ }^{1,46}$ J. C. Chen, ${ }^{1}$ M. L. Chen, ${ }^{1,42}$ P. L. Chen, ${ }^{53}$ S. J. Chen, ${ }^{33}$ X. R. Chen, ${ }^{30}$ Y. B. Chen, ${ }^{1,42}$ W. Cheng, ${ }^{55 c}$ X. K. Chu, ${ }^{35}$ G. Cibinetto, ${ }^{24 \mathrm{a}}$ F. Cossio, ${ }^{55 \mathrm{c}}$ H. L. Dai, ${ }^{1,42}$ J. P. Dai, ${ }^{37, \mathrm{~h}}$ A. Dbeyssi, ${ }^{15}$ D. Dedovich ${ }^{27}$ Z. Y. Deng, ${ }^{1}$ A. Denig, ${ }^{26}$ I. Denysenko, ${ }^{27}$ M. Destefanis, ${ }^{55,55 c}$ F. De Mori,${ }^{55 a, 55 c}$ Y. Ding, ${ }^{31}$ C. Dong, ${ }^{34}$ J. Dong, ${ }^{1,42}$ L. Y. Dong, ${ }^{1,46}$ M. Y. Dong, ${ }^{1,42,46}$ Z. L. Dou, ${ }^{33}$ S. X. Du,${ }^{60}$ P. F. Duan, ${ }^{1}$ J. Fang, ${ }^{1,42}$ S. S. Fang, ${ }^{1,46}$ Y. Fang, ${ }^{1}$ R. Farinelli, ${ }^{24 a, 24 b}$ L. Fava, ${ }^{55,55 c}$ S. Fegan, ${ }^{26}$ F. Feldbauer, ${ }^{4}$ G. Felici, ${ }^{23 a}$ C. Q. Feng, ${ }^{52,42}$ E. Fioravanti, ${ }^{24 a}$ M. Fritsch, ${ }^{4}$ C. D. Fu, ${ }^{1}$ Q. Gao, ${ }^{1}$ X. L. Gao,${ }^{52,42}$ Y. Gao, ${ }^{44}$ Y. G. Gao, ${ }^{6}$ Z. Gao, ${ }^{52,42}$ B. Garillon, ${ }^{26}$ I. Garzia, ${ }^{24 a}$ A. Gilman, ${ }^{49}$ K. Goetzen, ${ }^{11}$ L. Gong, ${ }^{34}$ W. X. Gong, ${ }^{1,42}$ W. Gradl, ${ }^{26}$ M. Greco, ${ }^{55 a, 55 \mathrm{c}}$ L. M. Gu,${ }^{33}$ M. H. Gu,${ }^{1,42}$ Y. T. Gu, ${ }^{13}$ A. Q. Guo, ${ }^{1}$ L. B. Guo, ${ }^{32}$ R. P. Guo, ${ }^{1,46}$ Y. P. Guo, ${ }^{26}$ A. Guskov ${ }^{27}$ Z. Haddadi, ${ }^{29}$ S. Han,${ }^{57}$ X. Q. Hao,${ }^{16}$ F. A. Harris,${ }^{47}$ K. L. He, ${ }^{1,46}$ X. Q. He ${ }^{51}$ F. H. Heinsius, ${ }^{4}$ T. Held, ${ }^{4}$ Y. K. Heng,${ }^{1,42,46}$ Z. L. Hou, ${ }^{1}$ H. M. Hu, ${ }^{1,46}$ J. F. Hu, ${ }^{37, h}$ T. Hu, ${ }^{1,42,46}$ Y. Hu, ${ }^{1}$ G. S. Huang,${ }^{52,42}$ J. S. Huang, ${ }^{16}$ X. T. Huang,${ }^{36}$ X. Z. Huang ${ }^{33}$

Z. L. Huang, ${ }^{31}$ T. Hussain, ${ }^{54}$ W. Ikegami Andersson, ${ }^{56}$ M. Irshad,${ }^{52,42}$ Q. Ji, ${ }^{1}$ Q. P. Ji ${ }^{16}$ X. B. Ji, ${ }^{1,46}$ X. L. Ji, ${ }^{1,42}$

X. S. Jiang, ${ }^{1,42,46}$ X. Y. Jiang, ${ }^{34}$ J. B. Jiao, ${ }^{36}$ Z. Jiao, ${ }^{18}$ D. P. Jin, ${ }^{1,42,46}$ S. Jin, ${ }^{1,46}$ Y. Jin, ${ }^{48}$ T. Johansson, ${ }^{56}$ A. Julin, ${ }^{49}$ N. Kalantar-Nayestanaki, ${ }^{29}$ X. S. Kang, ${ }^{34}$ M. Kavatsyuk, ${ }^{29}$ B. C. Ke, ${ }^{1}$ I. K. Keshk, ${ }^{4}$ T. Khan,${ }^{52,42}$ A. Khoukaz, ${ }^{50}$ P. Kiese, ${ }^{26}$ R. Kiuchi, ${ }^{1}$ R. Kliemt, ${ }^{11}$ L. Koch ${ }^{28}$ O. B. Kolcu, ${ }^{45 b, f}$ B. Kopf,${ }^{4}$ M. Kornicer, ${ }^{47}$ M. Kuemmel, ${ }^{4}$ M. Kuessner, ${ }^{4}$ A. Kupsc, ${ }^{56}$ M. Kurth, ${ }^{1}$ W. Kühn, ${ }^{28}$ J. S. Lange, ${ }^{28}$ P. Larin, ${ }^{15}$ L. Lavezzi, ${ }^{55 c}$ S. Leiber, ${ }^{4}$ H. Leithoff, ${ }^{26}$ C. Li,${ }^{56}$ Cheng Li, ${ }^{52,42}$ D. M. Li ${ }^{60}$ F. Li, ${ }^{1,42}$ F. Y. Li ${ }^{35}$ G. Li ${ }^{1}{ }^{1}$ H. B. Li ${ }^{1,46}$ H. J. Li, ${ }^{1,46}$ J. C. Li, ${ }^{1}$ J. W. Li ${ }^{40}$ K. J. Li, ${ }^{43}$ Kang Li, ${ }^{14}$ Ke Li, ${ }^{1}{ }^{2 e i ~ L i},{ }^{3}$ P. L. Li ${ }^{52,42}$ P. R. Li, ${ }^{46,7}$ Q. Y. Li ${ }^{36}$ T. Li, ${ }^{36}$ W. D. Li, ${ }^{1,46}$ W. G. Li, ${ }^{1}$ X. L. Li, ${ }^{36}$ X. N. Li, ${ }^{1,42}$ X. Q. Li, ${ }^{34}$ Z. B. Li, ${ }^{43}$ H. Liang, ${ }^{52,42}$ Y. F. Liang, ${ }^{39}$ Y. T. Liang, ${ }^{28}$ G. R. Liao, ${ }^{12}$ L. Z. Liao, ${ }^{1,46}$ J. Libby, ${ }^{21}$ C. X. Lin ${ }^{43}$ D. X. Lin,${ }^{15}$ B. Liu, ${ }^{37, h}$ B. J. Liu, ${ }^{1}$ C. X. Liu, ${ }^{1}$ D. Liu, ${ }^{52,42}$ D. Y. Liu, ${ }^{37, h}$ F. H. Liu, ${ }^{38}$ Fang Liu, ${ }^{1}$ Feng Liu, ${ }^{6}$ H. B. Liu, ${ }^{13}$ H. L. Liu, ${ }^{41}$ H. M. Liu, ${ }^{1,46}$ Huanhuan Liu, ${ }^{1}$ Huihui Liu, ${ }^{17}$ J. B. Liu, ${ }^{52,42}$ J. Y. Liu, ${ }^{1,46}$ K. Y. Liu, ${ }^{31}$ Ke Liu, ${ }^{6}$ L. D. Liu, ${ }^{35}$ Q. Liu, ${ }^{46}$ S. B. Liu, ${ }^{52,42}$ X. Liu, ${ }^{30}$ Y. B. Liu,${ }^{34}$ Z. A. Liu, ${ }^{1,42,46}$ Zhiqing Liu, ${ }^{26}$ Y. F. Long, ${ }^{35}$ X. C. Lou, ${ }^{1,42,46}$ H. J. Lu, ${ }^{18}$ J. G. Lu, ${ }^{1,42}$ Y. Lu, ${ }^{1}$ Y. P. Lu, ${ }^{1,42}$ C. L. Luo, ${ }^{32}$ M. X. Luo, ${ }^{59}$ T. Luo, ${ }^{9, j}$ X. L. Luo, ${ }^{1,42}$ S. Lusso, ${ }^{55 c}$ X. R. Lyu, ${ }^{46}$ F. C. Ma, ${ }^{31}$ H. L. Ma, ${ }^{1}$ L. L. Ma, ${ }^{36}$ M. M. Ma, ${ }^{1,46}$ Q. M. Ma, ${ }^{1}$ T. Ma, ${ }^{1}$ X. N. Ma, ${ }^{34}$ X. Y. Ma, ${ }^{1,42}$ Y. M. Ma, ${ }^{36}$ F. E. Maas, ${ }^{15}$ M. Maggiora,${ }^{55 a, 55 c}$ S. Maldaner, ${ }^{26}$ Q. A. Malik, ${ }^{54}$ A. Mangoni, ${ }^{23 \mathrm{~b}}$ Y. J. Mao, ${ }^{35}$ Z. P. Mao, ${ }^{1}$ S. Marcello, ${ }^{55 a, 55 \mathrm{c}}$ Z. X. Meng, ${ }^{48}$ J. G. Messchendorp, ${ }^{29}$ G. Mezzadri, ${ }^{24 \mathrm{~b}}$ J. Min, ${ }^{1,42}$ T. J. Min, ${ }^{33}$ R. E. Mitchell, ${ }^{22}$ X. H. Mo ${ }^{1,42,46}$ Y. J. Mo, ${ }^{6}$ C. Morales Morales, ${ }^{15}$ N. Yu. Muchnoi, ${ }^{10, d}$ H. Muramatsu, ${ }^{49}$ A. Mustafa, ${ }^{4}$ S. Nakhoul, ${ }^{11, \mathrm{~g}}$ Y. Nefedov, ${ }^{27}$ F. Nerling, ${ }^{11}$ I. B. Nikolaev, ${ }^{10, \mathrm{~d}}$ Z. Ning, ${ }^{1,42}$ S. Nisar, ${ }^{8}$ S. L. Niu, ${ }^{1,42}$ X. Y. Niu,${ }^{1,46}$ S. L. Olsen, ${ }^{46, k}$ Q. Ouyang, ${ }^{1,42,46}$ S. Pacetti, ${ }^{23 b}$ Y. Pan,${ }^{52,42}$ M. Papenbrock, ${ }^{56}$ P. Patteri, ${ }^{23 \mathrm{a}}$ M. Pelizaeus, ${ }^{4}$ J. Pellegrino, ${ }^{55 a, 55 \mathrm{c}}$ H. P. Peng ${ }^{52,42}$ Z. Y. Peng, ${ }^{13}$ K. Peters,${ }^{11, g}$ J. Pettersson, ${ }^{56}$ J. L. Ping, ${ }^{32}$ R. G. Ping, ${ }^{1,46}$ A. Pitka, ${ }^{4}$ R. Poling, ${ }^{49}$ V. Prasad,${ }^{5,42}$ H. R. Qi ${ }^{2}$ M. Qi ${ }^{33}$ T. Y. Qi, ${ }^{2}$ S. Qian, ${ }^{1,42}$ C. F. Qiao, ${ }^{46}$ N. Qin, ${ }^{57}$ X. S. Qin, ${ }^{4}$ Z. H. Qin, ${ }^{1,42}$ J. F. Qiu, ${ }^{1}$ S. Q. Qu ${ }^{34}$

K. H. Rashid, ${ }^{54, \mathrm{i}}$ C. F. Redmer, ${ }^{26}$ M. Richter, ${ }^{4}$ M. Ripka, ${ }^{26}$ A. Rivetti,${ }^{55 \mathrm{c}}$ M. Rolo, ${ }^{55 \mathrm{c}}$ G. Rong, ${ }^{1,46}$ Ch. Rosner, ${ }^{15}$ A. Sarantsev, ${ }^{27, \mathrm{e}}$ M. Savrié, ${ }^{24 b}$ K. Schoenning, ${ }^{56}$ W. Shan, ${ }^{19}$ X. Y. Shan,${ }^{52,42}$ M. Shao, ${ }^{52,42}$ C. P. Shen, ${ }^{2}$ P. X. Shen ${ }^{34}$ X. Y. Shen,${ }^{1,46}$ H. Y. Sheng, ${ }^{1}$ X. Shi, ${ }^{1,42}$ J. J. Song, ${ }^{36}$ W. M. Song, ${ }^{36}$ X. Y. Song, ${ }^{1}$ S. Sosio, ${ }^{55 a, 55 c}$ C. Sowa, ${ }^{4}$ S. Spataro, ${ }^{55 a, 55 c}$ G. X. Sun, ${ }^{1}$ J. F. Sun, ${ }^{16}$ L. Sun, ${ }^{57}$ S. S. Sun, ${ }^{1,46}$ X. H. Sun, ${ }^{1}$ Y. J. Sun, ${ }^{52,42}$ Y. K. Sun, ${ }^{52,42}$ Y. Z. Sun, ${ }^{1}$ Z. J. Sun, ${ }^{1,42}$ Z. T. Sun, ${ }^{1}$ Y. T. Tan, ${ }^{52,42}$ C. J. Tang, ${ }^{39}$ G. Y. Tang, ${ }^{1}$ X. Tang, ${ }^{1}$ M. Tiemens, ${ }^{29}$ B. Tsednee, ${ }^{25}$ I. Uman, ${ }^{45 d}$ B. Wang, ${ }^{1}$ B. L. Wang, ${ }^{46}$ C. W. Wang, ${ }^{33}$ D. Wang, ${ }^{35}$ D. Y. Wang, ${ }^{35}$ Dan Wang, ${ }^{46}$ K. Wang, ${ }^{1,42}$ L. L. Wang, ${ }^{1}$ L. S. Wang, ${ }^{1}$ M. Wang, ${ }^{36}$ Meng Wang, ${ }^{1,46}$ P. Wang, ${ }^{1}$ P. L. Wang, ${ }^{1}$ W. P. Wang, ${ }^{52,42}$ X. F. Wang, ${ }^{1}$ Y. Wang, ${ }^{52,42}$ Y. F. Wang, ${ }^{1,42,46}$ Z. Wang, ${ }^{1,42}$ Z. G. Wang, ${ }^{1,42}$ Z. Y. Wang, ${ }^{1}$ Zongyuan Wang, ${ }^{1,46}$ T. Weber, ${ }^{4}$ D. H. Wei, ${ }^{12}$ P. Weidenkaff, ${ }^{26}$ S. P. Wen, ${ }^{1}$ U. Wiedner, ${ }^{4}$ M. Wolke, ${ }^{56}$ L. H. Wu, ${ }^{1}$ L. J. Wu, ${ }^{1,46}$ Z. Wu, ${ }^{1,42}$ L. Xia, ${ }^{52,42}$ X. Xia ${ }^{36}$ Y. Xia, ${ }^{20}$ D. Xiao, ${ }^{1}$ Y. J. Xiao, ${ }^{1,46}$ Z. J. Xiao, ${ }^{32}$ Y. G. Xie, ${ }^{1,42}$ Y. H. Xie, ${ }^{6}$ X. A. Xiong, ${ }^{1,46}$ Q. L. Xiu,${ }^{1,42}$ G. F. Xu ${ }^{1}$ J. J. Xu,${ }^{1,46}$ L. Xu, ${ }^{1}$ Q. J. Xu,${ }^{14}$ X. P. Xu,${ }^{40}$ F. Yan, ${ }^{53}$ L. Yan, ${ }^{55 a, 55 c}$ W. B. Yan, ${ }^{52,42}$ W. C. Yan, ${ }^{2}$ Y. H. Yan, ${ }^{20}$ H. J. Yang, ${ }^{37, h}$ H. X. Yang, ${ }^{1}$ L. Yang, ${ }^{57}$ R. X. Yang, ${ }^{52,42}$ Y. H. Yang, ${ }^{33}$ Y. X. Yang ${ }^{12}$ Yifan Yang, ${ }^{1,46}$ Z. Q. Yang, ${ }^{20}$ M. Ye ${ }^{1,42}$ M. H. Ye, ${ }^{7}$ J. H. Yin, ${ }^{1}$ Z. Y. You, ${ }^{43}$ B. X. Yu, ${ }^{1,42,46}$ C. X. Yu, ${ }^{34}$ J. S. Yu, ${ }^{30}$ J. S. Yu, ${ }^{20}$ C. Z. Yuan, ${ }^{1,46}$ Y. Yuan, ${ }^{1}$ A. Yuncu, ${ }^{45 b, a}$ A. A. Zafar, ${ }^{54}$ Y. Zeng, ${ }^{20}$ B. X. Zhang, ${ }^{1}$ B. Y. Zhang, ${ }^{1,42}$ C. C. Zhang, ${ }^{1}$ D. H. Zhang, ${ }^{1}$ H. H. Zhang, ${ }^{43}$ H. Y. Zhang, ${ }^{1,42}$ J. Zhang, ${ }^{1,46}$ J. L. Zhang, ${ }^{58}$ J. Q. Zhang, ${ }^{4}$ J. W. Zhang, ${ }^{1,42,46}$ J. Y. Zhang, ${ }^{1}$ J. Z. Zhang, ${ }^{1,46}$ K. Zhang, ${ }^{1,46}$ L. Zhang, ${ }^{44}$ S. F. Zhang, ${ }^{33}$ T. J. Zhang, ${ }^{37, h}$ X. Y. Zhang, ${ }^{36}$ Y. Zhang, ${ }^{52,42}$ Y. H. Zhang, ${ }^{1,42}$ Y. T. Zhang, ${ }^{52,42}$ Yang Zhang, ${ }^{1}$ Yao Zhang, ${ }^{1}$ Yu Zhang, ${ }^{46}$ Z. H. Zhang, ${ }^{6}$ Z. P. Zhang, ${ }^{52}$ Z. Y. Zhang, ${ }^{57}$ G. Zhao, ${ }^{1}$ J. W. Zhao, ${ }^{1,42}$ J. Y. Zhao, ${ }^{1,46}$ 
J. Z. Zhao, ${ }^{1,42}$ Lei Zhao, ${ }^{52,42}$ Ling Zhao, ${ }^{1}$ M. G. Zhao, ${ }^{34}$ Q. Zhao, ${ }^{1}$ S. J. Zhao, ${ }^{60}$ T. C. Zhao, ${ }^{1}$ Y. B. Zhao, ${ }^{1,42}$ Z. G. Zhao,${ }^{52,42}$ A. Zhemchugov, ${ }^{27, b}$ B. Zheng, ${ }^{53}$ J. P. Zheng, ${ }^{1,42}$ W. J. Zheng, ${ }^{36}$ Y. H. Zheng, ${ }^{46}$ B. Zhong, ${ }^{32}$ L. Zhou, ${ }^{1,42}$ Q. Zhou, ${ }^{1,46}$ X. Zhou, ${ }^{57}$ X. K. Zhou, ${ }^{52,42}$ X. R. Zhou, ${ }^{52,42}$ X. Y. Zhou, ${ }^{1}$ Xiaoyu Zhou, ${ }^{20}$ Xu Zhou, ${ }^{20}$ A. N. Zhu, ${ }^{1,46}$ J. Zhu, ${ }^{34}$ J. Zhu ${ }^{43}$ K. Zhu, ${ }^{1}$ K. J. Zhu, ${ }^{1,42,46}$ S. Zhu, ${ }^{1}$ S. H. Zhu, ${ }^{51}$ X. L. Zhu, ${ }^{44}$ Y. C. Zhu, ${ }^{52,42}$ Y. S. Zhu, ${ }^{1,46}$ Z. A. Zhu, ${ }^{1,46}$ J. Zhuang, ${ }^{1,42}$ B. S. Zou, ${ }^{1}$ and J.H. Zou ${ }^{1}$

\title{
(BESIII Collaboration)
}

\author{
${ }^{1}$ Institute of High Energy Physics, Beijing 100049, People's Republic of China \\ ${ }^{2}$ Beihang University, Beijing 100191, People's Republic of China \\ ${ }^{3}$ Beijing Institute of Petrochemical Technology, Beijing 102617, People's Republic of China \\ ${ }^{4}$ Bochum Ruhr-University, D-44780 Bochum, Germany \\ ${ }^{5}$ Carnegie Mellon University, Pittsburgh, Pennsylvania 15213, USA \\ ${ }^{6}$ Central China Normal University, Wuhan 430079, People's Republic of China \\ ${ }^{7}$ China Center of Advanced Science and Technology, Beijing 100190, People's Republic of China \\ ${ }^{8}$ COMSATS Institute of Information Technology, Lahore, Defence Road, Off Raiwind Road, 54000 Lahore, Pakistan \\ ${ }^{9}$ Fudan University, Shanghai 200443, People's Republic of China \\ ${ }^{10}$ G.I. Budker Institute of Nuclear Physics SB RAS (BINP), Novosibirsk 630090, Russia \\ ${ }^{11}$ GSI Helmholtzcentre for Heavy Ion Research GmbH, D-64291 Darmstadt, Germany \\ ${ }^{12}$ Guangxi Normal University, Guilin 541004, People's Republic of China \\ ${ }^{13}$ Guangxi University, Nanning 530004, People's Republic of China \\ ${ }^{14}$ Hangzhou Normal University, Hangzhou 310036, People's Republic of China \\ ${ }^{15}$ Helmholtz Institute Mainz, Johann-Joachim-Becher-Weg 45, D-55099 Mainz, Germany \\ ${ }^{16}$ Henan Normal University, Xinxiang 453007, People's Republic of China \\ ${ }^{17}$ Henan University of Science and Technology, Luoyang 471003, People's Republic of China \\ ${ }^{18}$ Huangshan College, Huangshan 245000, People's Republic of China \\ ${ }^{19}$ Hunan Normal University, Changsha 410081, People's Republic of China \\ ${ }^{20}$ Hunan University, Changsha 410082, People's Republic of China \\ ${ }^{21}$ Indian Institute of Technology Madras, Chennai 600036, India \\ ${ }^{22}$ Indiana University, Bloomington, Indiana 47405, USA \\ ${ }^{23 a}$ INFN Laboratori Nazionali di Frascati, I-00044 Frascati, Italy \\ ${ }^{23 \mathrm{~b}}$ INFN and University of Perugia, I-06100 Perugia, Italy \\ ${ }^{24 a}$ INFN Sezione di Ferrara, I-44122 Ferrara, Italy \\ ${ }^{24 \mathrm{~b}}$ University of Ferrara, I-44122 Ferrara, Italy \\ ${ }^{25}$ Institute of Physics and Technology, Peace Ave. 54B, Ulaanbaatar 13330, Mongolia \\ ${ }^{26}$ Johannes Gutenberg University of Mainz, Johann-Joachim-Becher-Weg 45, D-55099 Mainz, Germany \\ ${ }^{27}$ Joint Institute for Nuclear Research, 141980 Dubna, Moscow region, Russia \\ ${ }^{28}$ Justus-Liebig-Universitaet Giessen, II. Physikalisches Institut, Heinrich-Buff-Ring 16, D-35392 Giessen, Germany \\ ${ }^{29}$ KVI-CART, University of Groningen, NL-9747 AA Groningen, Netherlands \\ ${ }^{30}$ Lanzhou University, Lanzhou 730000, People's Republic of China \\ ${ }^{31}$ Liaoning University, Shenyang 110036, People's Republic of China \\ ${ }^{32}$ Nanjing Normal University, Nanjing 210023, People's Republic of China \\ ${ }^{33}$ Nanjing University, Nanjing 210093, People's Republic of China \\ ${ }^{34}$ Nankai University, Tianjin 300071, People's Republic of China \\ ${ }^{35}$ Peking University, Beijing 100871, People's Republic of China \\ ${ }^{36}$ Shandong University, Jinan 250100, People's Republic of China \\ ${ }^{37}$ Shanghai Jiao Tong University, Shanghai 200240, People's Republic of China \\ ${ }^{38}$ Shanxi University, Taiyuan 030006, People's Republic of China \\ ${ }^{39}$ Sichuan University, Chengdu 610064, People's Republic of China \\ ${ }^{40}$ Soochow University, Suzhou 215006, People's Republic of China \\ ${ }^{41}$ Southeast University, Nanjing 211100, People's Republic of China \\ ${ }^{42}$ State Key Laboratory of Particle Detection and Electronics, Beijing 100049, Hefei 230026, People's Republic of China \\ ${ }^{43}$ Sun Yat-Sen University, Guangzhou 510275, People's Republic of China \\ ${ }^{44}$ Tsinghua University, Beijing 100084, People's Republic of China \\ ${ }^{45 a}$ Ankara University, 06100 Tandogan, Ankara, Turkey \\ ${ }^{45 \mathrm{~b}}$ Istanbul Bilgi University, 34060 Eyup, Istanbul, Turkey \\ ${ }^{45 c}$ Uludag University, 16059 Bursa, Turkey \\ ${ }^{45 \mathrm{~d}}$ Near East University, Nicosia, North Cyprus, Mersin 10, Turkey \\ ${ }^{46}$ University of Chinese Academy of Sciences, Beijing 100049, People's Republic of China
}




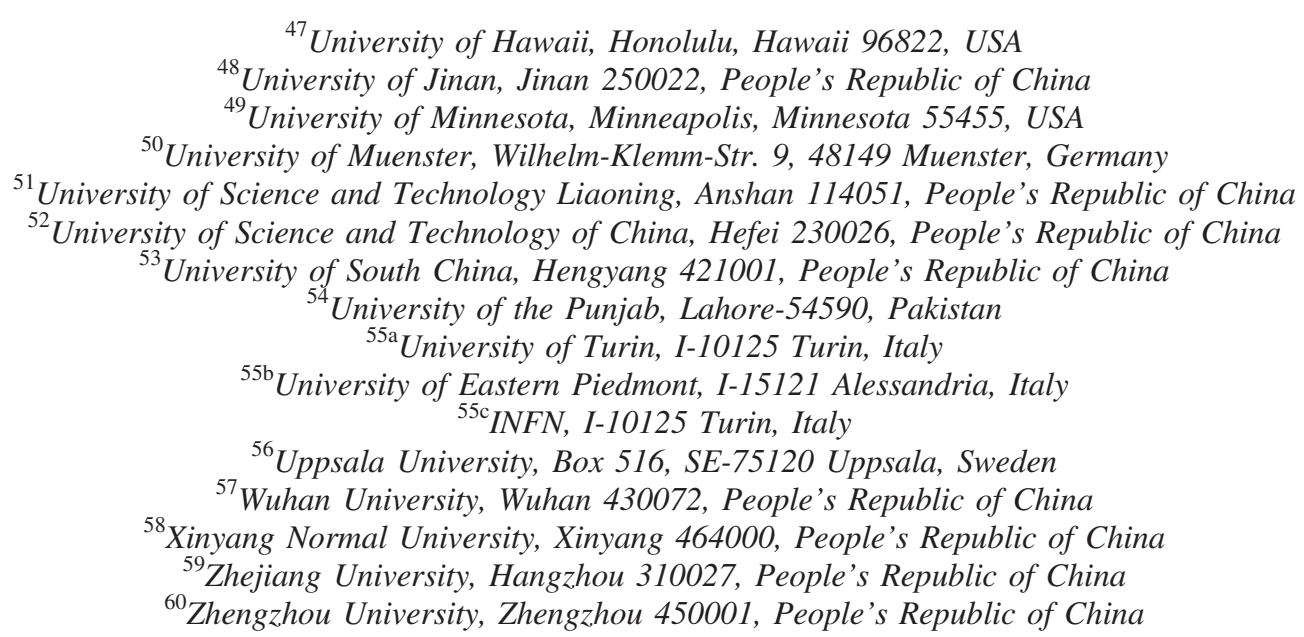

(Received 8 January 2019; revised manuscript received 27 February 2019; published 25 March 2019)

Using $e^{+} e^{-}$annihilation data corresponding to an integrated luminosity of $3.19 \mathrm{fb}^{-1}$ collected at a center-of-mass energy of $4.178 \mathrm{GeV}$ with the BESIII detector, we measure the absolute branching fractions $\mathcal{B}_{D_{s}^{+} \rightarrow \eta e^{+} \nu_{e}}=\left(2.323 \pm 0.063_{\text {stat }} \pm 0.063_{\text {syst }}\right) \%$ and $\mathcal{B}_{D_{s}^{+} \rightarrow \eta^{\prime} e^{+} \nu_{e}}=\left(0.824 \pm 0.073_{\text {stat }} \pm 0.027_{\text {syst }}\right) \%$ via a tagged analysis technique, where one $D_{s}$ is fully reconstructed in a hadronic mode. Combining these measurements with previous BESIII measurements of $\mathcal{B}_{D^{+} \rightarrow \eta^{(\prime)} e^{+} \nu_{e}}$, the $\eta-\eta^{\prime}$ mixing angle in the quark flavor basis is determined to be $\phi_{P}=\left(40.1 \pm 2.1_{\text {stat }} \pm 0.7_{\text {syst }}\right)^{\circ}$. From the first measurements of the dynamics of $D_{s}^{+} \rightarrow \eta^{(\prime)} e^{+} \nu_{e}$ decays, the products of the hadronic form factors $f_{+}^{\eta^{(\prime)}}(0)$ and the CabibboKobayashi-Maskawa matrix element $\left|V_{\mathrm{cs}}\right|$ are determined with different form factor parametrizations. For the two-parameter series expansion, the results are $f_{+}^{\eta}(0)\left|V_{c s}\right|=0.4455 \pm 0.0053_{\text {stat }} \pm 0.0044_{\text {syst }}$ and $f_{+}^{\eta^{\prime}}(0)\left|V_{\text {cs }}\right|=0.477 \pm 0.049_{\text {stat }} \pm 0.011_{\text {syst }}$.

DOI: 10.1103/PhysRevLett.122.121801

Exclusive $D$ semileptonic (SL) decays provide a powerful way to extract the weak and strong interaction couplings of quarks due to simple theoretical treatment [1-3]. In the standard model, the rate of $D_{s}^{+} \rightarrow \eta e^{+} \nu_{e}$ and $D_{s}^{+} \rightarrow \eta^{\prime} e^{+} \nu_{e}$ depends not only on $V_{\text {cs }}$, an element of the CabibboKobayashi-Maskawa (CKM) matrix describing weak transitions between the charm and strange quarks, but also on the dynamics of strong interaction, parametrized by the form factor $(\mathrm{FF}) f_{+}^{\eta^{(\prime)}}\left(q^{2}\right)$, where $q$ is the momentum transfer to the $e^{+} \nu_{e}$ system. Unlike the final-state hadrons $K$ and $\pi$, the mesons $\eta^{(\prime)}$ are especially intriguing because the spectator quark plays an important role in forming the final state. This gives access to the singlet-octet mixing of the $\eta-\eta^{\prime}$ gluon [4,5], whose mixing parameter can be determined from the SL decays, and, consequently, gives a deeper understanding of nonperturbative QCD confinement.

Published by the American Physical Society under the terms of the Creative Commons Attribution 4.0 International license. Further distribution of this work must maintain attribution to the author(s) and the published article's title, journal citation, and DOI. Funded by SCOAP.
Recently, the FF $f_{+}^{\eta^{(\prime)}}(0)$ were calculated using lattice quantum chromodynamics (LQCD) [6] and QCD lightcone sum rules (LCSR) [7,8] by assuming particular admixtures of quarks and gluons [9-11] for $\eta$ and $\eta^{\prime}$ mesons. As information concerning the gluon content in the $\eta^{\prime}$ remains inconclusive, large uncertainties may be involved. Measurements of $f_{+}^{\eta^{(\prime)}}(0)$ are crucial to calibrate these theoretical calculations. Once the predicted $f_{+}^{\eta^{(1)}}(0)$ pass these experimental tests, they will help determine $\left|V_{\text {cs }}\right|$, and, in return, help test the unitarity of the CKM quark mixing matrix. Additionally, measurements of the branching fractions (BFs) of $D_{s}^{+} \rightarrow$ $\eta^{(\prime)} e^{+} \nu_{e}$ can shed light on $\eta-\eta^{\prime}$-gluon mixing. The $\eta-\eta^{\prime}$ mixing angle in the quark flavor basis, $\phi_{P}$, can be related to the BFs of the $D$ and $D_{s}$ via $\cot ^{4} \phi_{P}=$ $\left\{\left[\left(\Gamma_{D_{s}^{+} \rightarrow \eta^{\prime} e^{+} \nu_{e}}\right) /\left(\Gamma_{D_{s}^{+} \rightarrow \eta e^{+} \nu_{e}}\right)\right] /\left[\left(\Gamma_{D^{+} \rightarrow \eta^{\prime} e^{+} \nu_{e}}\right) /\left(\Gamma_{D^{+} \rightarrow \eta e^{+} \nu_{e}}\right)\right]\right\}$, in which a possible gluon component cancels [9]. Determination of $\phi_{P}$ gives a complementary constraint on the role of gluonium in the $\eta^{\prime}$, thus helping to improve our understanding of nonperturbative QCD dynamics and benefiting theoretical calculations of $D$ and $B$ decays involving the $\eta^{(\prime)}$. 
Previous measurements of the BFs of $D_{s}^{+} \rightarrow \eta^{(\prime)} e^{+} \nu_{e}$ were made by CLEO [12-14] and BESIII [15], but these measurements include large uncertainties. This Letter reports improved measurements of the BFs and the first experimental studies of the dynamics of $D_{s}^{+} \rightarrow \eta^{(\prime)} e^{+} \nu_{e}$ [16]. Based on these, the first measurements of $f_{+}^{\eta^{(\prime)}}(0)$ are made, and measurements of $\left|V_{\mathrm{cs}}\right|$ and $\phi_{P}$ are presented.

This analysis is performed using $e^{+} e^{-}$collision data corresponding to an integrated luminosity of $3.19 \mathrm{fb}^{-1}$ taken at a center-of-mass energy $E_{\text {c.m. }}=4.178 \mathrm{GeV}$ with the BESIII detector. A description of the design and performance of the BESIII detector can be found in Ref. [17]. For the data used in this Letter, the end cap time-of-flight system was upgraded with multigap resistive plate chambers with a time resolution of 60 ps $[18,19]$. Monte Carlo (MC) simulated events are generated with a GEANT4-based [20] detector simulation software package, which includes the geometric description and a simulation of the response of the detector. An inclusive MC sample with equivalent luminosity 35 times that of data is produced at $E_{\text {c.m. }}=4.178 \mathrm{GeV}$. It includes open charm processes, initial state radiation (ISR) production of $\psi(3770)$, $\psi(3686)$, and $J / \psi, q \bar{q}(q=u, d, s)$ continuum processes, along with Bhabha scattering, $\mu^{+} \mu^{-}, \tau^{+} \tau^{-}$, and $\gamma \gamma$ events. The open charm processes are generated using CONEXC [21]. The effects of ISR and final state radiation (FSR) are considered. The known particle decays are generated with the BFs taken from the Particle Data Group (PDG) [22] by EVTGEN [23], and the other modes are generated using LUNDCHARM [24]. The SL decays $D_{s}^{+} \rightarrow \eta^{(\prime)} e^{+} \nu_{e}$ are simulated with the modified pole model [25].

At $E_{\text {c.m. }}=4.178 \mathrm{GeV}, D_{s}^{+}$mesons are produced mainly from the processes $e^{+} e^{-} \rightarrow D_{s}^{+} D_{s}^{*-}+$ c.c. $\rightarrow D_{s}^{+} \gamma\left(\pi^{0}\right) D_{s}^{-}$. We first fully reconstruct one $D_{s}^{-}$in one of several hadronic decay modes [called the single-tag (ST) $D_{s}^{-}$]. We then examine the SL decays of the $D_{s}^{+}$and the $\gamma\left(\pi^{0}\right)$ from the $D_{s}^{*}$ [called double-tag (DT) $D_{s}^{+}$]. The BF of the SL decay is determined by

$$
\mathcal{B}_{\mathrm{SL}}=N_{\mathrm{DT}}^{\mathrm{tot}} /\left(N_{\mathrm{ST}}^{\mathrm{tot}} \times \epsilon_{\gamma\left(\pi^{0}\right) \mathrm{SL}}\right),
$$

where $N_{\mathrm{ST}}^{\mathrm{tot}}$ and $N_{\mathrm{DT}}^{\mathrm{tot}}$ are the ST and DT yields in data, $\epsilon_{\gamma\left(\pi^{0}\right) \mathrm{SL}}$ is the efficiency of finding $\gamma\left(\pi^{0}\right) \eta^{(\prime)} e^{+} \nu_{e}$ determined by $\sum_{k}\left(N_{\mathrm{ST}}^{k} / N_{\mathrm{ST}}^{\text {tot }}\right)\left(\epsilon_{\mathrm{DT}}^{k} / \epsilon_{\mathrm{ST}}^{k}\right)$, where $\epsilon_{\mathrm{ST}}^{k}$ and $\epsilon_{\mathrm{DT}}^{k}$ are the efficiencies of selecting ST and DT candidates in the $k$ th tag mode, and estimated by analyzing the inclusive MC sample and the independent signal MC events of various DT modes, respectively.

The ST $D_{s}^{-}$candidates are reconstructed using fourteen hadronic decay modes as shown in Fig. 1. The selection criteria for charged tracks and $K_{S}^{0}$, and the particle identification (PID) requirements for $\pi^{ \pm}$and $K^{ \pm}$, are the same as those used in Ref. [26]. Positron PID is performed by using the specific ionization energy loss in the main drift

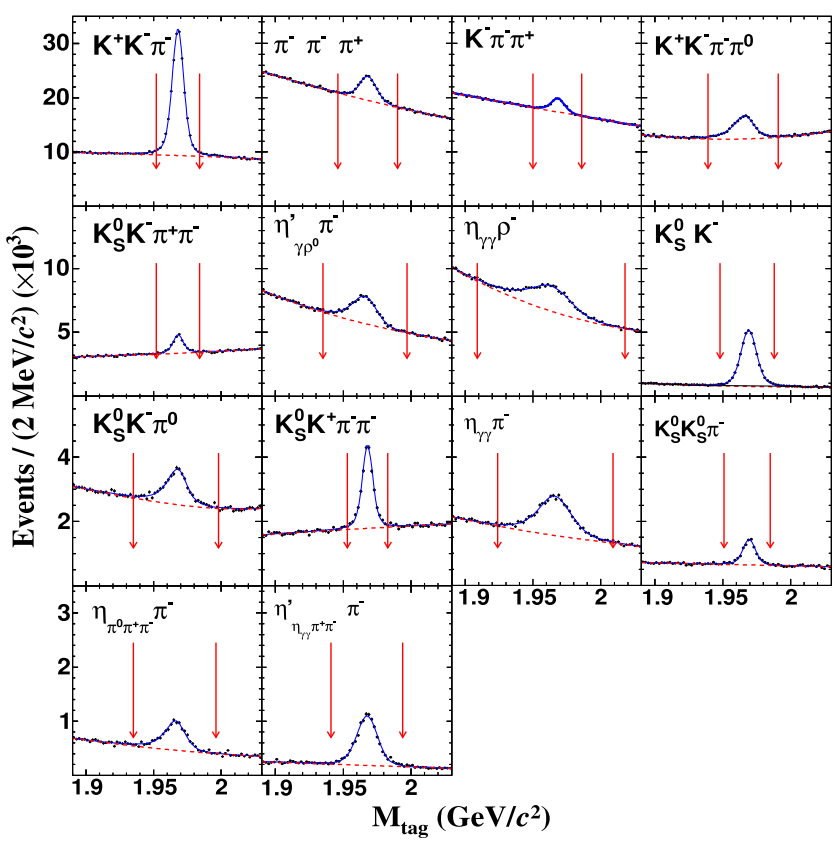

FIG. 1. Spectra of $M_{\text {tag }}$ of the ST candidates. Dots with error bars are data. Blue solid curves are the fit results. Dashed curves are the fitted backgrounds. The black solid curve in the $K_{S}^{0} K^{-}$ mode is $D^{-} \rightarrow K_{S}^{0} \pi^{-}$background. Pairs of arrows denote the $D_{s}^{-}$ signal regions within $\pm 3 \sigma$ around the nominal $D_{s}^{-}$mass [22].

chamber, the time of flight, and the energy deposited in the electromagnetic calorimeter (EMC). Confidence levels for the pion, kaon, and positron hypotheses $\left(\mathcal{L}_{\pi}, \mathcal{L}_{K}\right.$, and $\left.\mathcal{L}_{e}\right)$ are formed. Positron candidates must satisfy $\mathcal{L}_{e}>0.001$ and $\mathcal{L}_{e} /\left(\mathcal{L}_{e}+\mathcal{L}_{\pi}+\mathcal{L}_{K}\right)>0.8$. The energy loss of the positron due to bremsstrahlung is partially recovered by adding the energies of the EMC showers that are within $10^{\circ}$ of the positron direction and not matched to other particles (FSR recovery).

Photon candidates are selected from the EMC showers that begin within $700 \mathrm{~ns}$ of the event start time and have an energy greater than 25 (50) $\mathrm{MeV}$ in the barrel (end cap) region of the EMC [17]. Candidates of $\pi^{0}$ or $\eta_{\gamma \gamma}$ are formed by photon pairs with an invariant mass in the range $(0.115$, $0.150)$ or $(0.50,0.57) \mathrm{GeV} / c^{2}$. To improve the momentum resolution, the $\gamma \gamma$ invariant mass is constrained to the $\pi^{0}$ or $\eta$ nominal mass [22] via a kinematic fit. Candidates of $\eta_{\pi^{0} \pi^{+} \pi^{-}}, \eta_{\eta_{\gamma \gamma} \pi^{+} \pi^{-}}^{\prime}, \eta_{\gamma \rho^{0}}^{\prime}, \rho^{0}$, and $\rho^{-}$are formed from $\pi^{+} \pi^{-} \pi^{0}$, $\eta_{\gamma \gamma} \pi^{+} \pi^{-}, \gamma \rho_{\pi^{+} \pi^{-}}^{0}, \pi^{+} \pi^{-}$, and $\pi^{-} \pi^{0}$ combinations whose invariant masses fall in the ranges $(0.53,0.57)$, $(0.946,0.970), \quad(0.940,0.976),(0.57,0.97)$, and $(0.57$, $0.97) \mathrm{GeV} / c^{2}$, respectively.

To remove soft pions originating from $D^{*}$ transitions, the momenta of pions from the ST $D_{s}^{-}$are required to be larger than $0.1 \mathrm{GeV} / c$. For the tag modes $D_{s}^{-} \rightarrow \pi^{+} \pi^{-} \pi^{-}$and $K^{-} \pi^{+} \pi^{-}$, the contributions of $D_{s}^{-} \rightarrow K_{S}^{0} \pi^{-}$and $K_{S}^{0} K^{-}$are removed by requiring $M_{\pi^{+} \pi^{-}}$outside $\pm 0.03 \mathrm{GeV} / c^{2}$ around the $K_{S}^{0}$ nominal mass [22]. 
The ST $D_{s}^{-}$mesons are identified by the beam constrained mass $M_{\mathrm{BC}} \equiv \sqrt{\left(E_{\mathrm{c} . \mathrm{m} .} / 2\right)^{2}-\left|\vec{p}_{D_{s}^{-}}\right|^{2}}$ and the $D_{s}^{-}$recoil mass $M_{\text {rec }} \equiv \sqrt{\left(E_{\mathrm{c} . \mathrm{m} .}-\sqrt{\left|\vec{p}_{D_{s}^{-}}\right|^{2}+M_{D_{s}^{-}}^{2}}\right)^{2}-\left|\vec{p}_{D_{s}^{-}}\right|^{2}}$, where $\vec{p}_{D_{s}^{-}}$ is the 3-momentum of the ST candidate and $M_{D_{s}^{-}}$is the nominal $D_{s}^{-}$mass [22]. Non- $D_{s}^{+} D_{s}^{*-}$ events are suppressed by requiring $M_{\mathrm{BC}} \in(2.010,2.073) \mathrm{GeV} / c^{2}$. In each event, only the candidate with $M_{\text {rec }}$ closest to the nominal $D_{s}^{*+}$ mass [22] is chosen. The ST yield is determined by fits to the $M_{\text {tag }}$ spectra for each of the 14 tag modes shown in Fig. 1, where $M_{\text {tag }}$ is the invariant mass of the ST candidate. Signals and the $D^{-} \rightarrow K_{S}^{0} \pi^{-}$peaking background in the $D_{s}^{-} \rightarrow K_{S}^{0} K^{-}$mode are described by MC-simulated shapes. The nonpeaking background is modeled by a second- or third-order Chebychev polynomial. To account for the resolution difference between data and MC simulation, the MC simulated shape(s) is convolved with a Gaussian for each tag mode. The reliability of the fitted nonpeaking background has been verified using the inclusive $\mathrm{MC}$ sample. Events in the signal regions, denoted by the boundaries in each subfigure of Fig. 1, are kept for further analysis. The total ST yield is $N_{\mathrm{ST}}^{\text {tot }}=395142 \pm 1923$.

Once the $D_{s}^{-}$tag has been found, the photon or $\pi^{0}$ from the $D_{s}^{*+}$ transition is selected. We define the energy difference $\Delta E \equiv E_{\mathrm{c} . \mathrm{m} .}-E_{\mathrm{tag}}-E_{\gamma\left(\pi^{0}\right)+D_{s}^{-}}^{\mathrm{rec}}-E_{\gamma\left(\pi^{0}\right)}$, where $E_{\gamma\left(\pi^{0}\right)+D_{s}^{-}}^{\mathrm{rec}} \equiv \sqrt{\left|-\vec{p}_{\gamma\left(\pi^{0}\right)}-\vec{p}_{\mathrm{tag}}\right|^{2}+M_{D_{s}^{+}}^{2}}, E_{i}$ and $\vec{p}_{i}$ $\left[i=\gamma\left(\pi^{0}\right)\right.$ or tag $]$ are the energy and momentum of $\gamma\left(\pi^{0}\right)$ or $D_{s}^{-}$tag, respectively. All unused $\gamma$ or $\pi^{0}$ candidates are looped over and that with the minimum $|\Delta E|$ is chosen. Candidates with $\Delta E \in(-0.04,0.04) \mathrm{GeV}$ are accepted. The signal candidates are examined by the kinematic variable $\quad \mathrm{MM}^{2} \equiv\left(E_{\mathrm{c} . \mathrm{m} .}-E_{\mathrm{tag}}-E_{\gamma\left(\pi^{0}\right)}-E_{\eta^{(\prime)}}-E_{e}\right)^{2}-$ $\left|-\vec{p}_{\text {tag }}-\vec{p}_{\gamma\left(\pi^{0}\right)}-\vec{p}_{\eta^{(\prime)}}-\vec{p}_{e}\right|^{2}$, where $E_{i}$ and $\vec{p}_{i}(i=e$ or $\left.\eta^{(\prime)}\right)$ are the energy and momentum of $e^{+}$or $\eta^{(\prime)}$. To suppress backgrounds from $D_{s}^{+}$hadronic decays, the maximum energy of the unused showers $\left(E_{\gamma e x t r a}^{\max }\right)$ must be less than $0.3 \mathrm{GeV}$ and events with additional charged tracks $\left(N_{\text {char }}^{\text {extra }}\right)$ are removed. We require $M_{\eta^{\prime} e^{+}}<1.9 \mathrm{GeV} / c^{2}$ for $D_{s}^{+} \rightarrow \eta^{\prime} e^{+} \nu_{e}$ and $\cos \theta_{\text {hel }} \in(-0.85,0.85)$ for $D_{s}^{+} \rightarrow$ $\eta_{\gamma \rho^{0}}^{\prime} e^{+} \nu_{e}$ to further suppress the $D_{s}^{+} \rightarrow \eta^{\prime} \pi^{+}$and $D_{s}^{+} \rightarrow$ $\phi e^{+} \nu_{e}$ backgrounds, where $\theta_{\text {hel }}$ is the helicity angle between the momentum directions of the $\pi^{+}$and the $\eta^{\prime}$ in the $\rho^{0}$ rest frame.

Figure 2 shows the $\mathrm{MM}^{2}$ distribution after all selection criteria have been applied. The signal yields are determined from a simultaneous unbinned maximum likelihood fit to these spectra, where $\mathcal{B}_{D_{s}^{+} \rightarrow \eta^{(\prime)} e^{+} \nu_{e}}$ measured using two different $\eta^{(\prime)}$ subdecays are constrained to be the same after considering the different efficiencies and subdecay BFs. The signal and background components in the fit are described by shapes derived from MC simulation. For

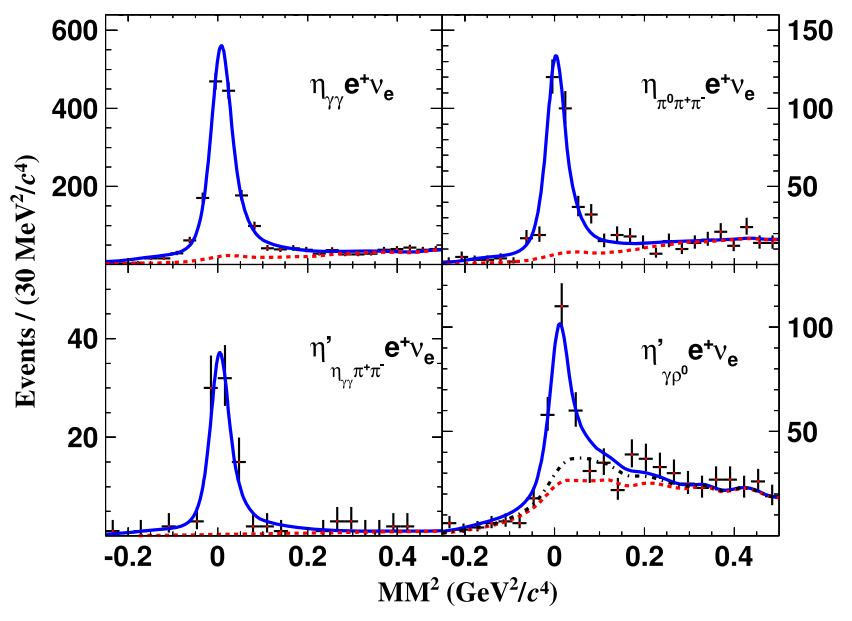

FIG. 2. Distributions of $\mathrm{MM}^{2}$ of the SL candidates. Dots with error bars are data. Solid curves are the best fits. Dotted curves are the fitted nonpeaking backgrounds. The dash-dotted curve is the peaking background due to $D_{s}^{+} \rightarrow \phi e^{+} \nu_{e}$.

the decay $D_{s}^{+} \rightarrow \eta_{\gamma \rho^{0}}^{\prime} e^{+} \nu_{e}$, some peaking background from $D_{s}^{+} \rightarrow \phi e^{+} \nu_{e}$ still remains. This background is modeled by a separate component in the fit; its size and shape are fixed based on MC simulation.

Table I summarizes the efficiencies for finding SL decays, the observed signal yields, and the obtained BFs.

With the DT method, the BF measurements are insensitive to the ST selection. The following relative systematic uncertainties in the BF measurements are assigned. The uncertainty in the ST yield is estimated to be $0.6 \%$ by alternative fits to the $M_{\text {tag }}$ spectra with different signal shapes, background parameters, and fit ranges. The uncertainties in the tracking or PID efficiencies are assigned as $0.5 \%$ per $\pi^{ \pm}$by studying $e^{+} e^{-} \rightarrow K^{+} K^{-} \pi^{+} \pi^{-}$, and $0.5 \%$ per $e^{+}$by radiative Bhabha process, respectively. The uncertainties of the $E_{\text {yextra }}^{\max }$ and $N_{\text {char }}^{\text {extra }}$ requirements are estimated to be $0.5 \%$ and $0.9 \%$ by analyzing DT hadronic events. The uncertainties of the $\Delta E$ requirement, FSR recovery and $\theta_{\text {hel }}$ requirement are estimated with and without each requirement, and the $\mathrm{BF}$ changes are $0.8 \%$, $0.8 \%$, and $0.1 \%$, respectively, which are taken as the

TABLE I. Efficiencies $\left(\epsilon_{\gamma\left(\pi^{0}\right) \mathrm{SL}}\right)$, signal yields $\left(N_{\mathrm{DT}}^{\text {tot }}\right)$, and the obtained BFs. Uncertainties on the least significant digits are shown in parentheses, where the first (second) uncertainties are statistical (systematic). The efficiencies do not include the BFs of $\eta^{(\prime)}$ subdecays.

\begin{tabular}{lcccc}
\hline \hline Decay & $\eta^{(\prime)}$ decay & $\epsilon_{\gamma\left(\pi^{0}\right) \mathrm{SL}}(\%)$ & $N_{\mathrm{DT}}^{\mathrm{tot}}$ & $\mathcal{B}_{\mathrm{SL}}(\%)$ \\
\hline$\eta e^{+} \nu_{e}$ & $\gamma \gamma$ & $41.11(27)$ & $1834(47)$ & $2.323(63)(63)$ \\
& $\pi^{0} \pi^{+} \pi^{-}$ & $16.06(31)$ & & \\
$\eta^{\prime} e^{+} \nu_{e}$ & $\eta \pi^{+} \pi^{-}$ & $14.07(10)$ & $261(22)$ & $0.824(73)(27)$ \\
& $\gamma \rho^{0}$ & $18.98(10)$ & & \\
\hline \hline
\end{tabular}




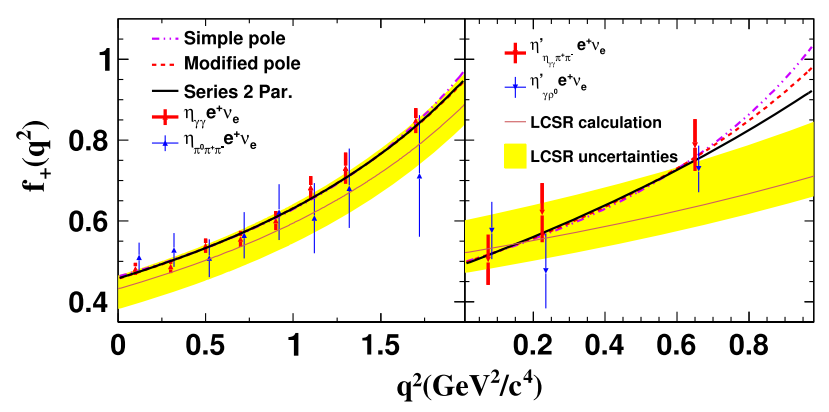

FIG. 3. Projections of the fits to $\Delta \Gamma_{\mathrm{msr}}^{i}$ of $D_{s}^{+} \rightarrow \eta^{(\prime)} e^{+} \nu_{e}$. Dots with error bars are data. The $\Delta \Gamma_{\mathrm{msr}}^{i} \mathrm{~s}$ measured with the two $\eta^{(\prime)}$ decay modes are offset horizontally for improved clarity. The curves show the best fits as described in text. Pink lines with yellow bands are the LCSR calculations with uncertainties [7].

individual uncertainties. The uncertainties of the selection of neutral particles are assigned as $1.0 \%$ per photon by studying $J / \psi \rightarrow \pi^{+} \pi^{-} \pi^{0}$ [27] and $1.0 \%$ per $\pi^{0}$ or $\eta$ by studying $e^{+} e^{-} \rightarrow K^{+} K^{-} \pi^{+} \pi^{-} \pi^{0}$. The uncertainty due to the signal model is estimated to be $0.5 \%$ by comparing the DT efficiencies before and after reweighting the $q^{2}$ distribution of the signal MC events to data. The uncertainty of the $\mathrm{MM}^{2}$ fit is assigned as $0.9 \%, 1.3 \%, 1.2 \%$, and $1.2 \%$ for $D_{s}^{+} \rightarrow \eta_{\gamma \gamma} e^{+} \nu_{e}, \eta_{\pi^{0} \pi^{+} \pi^{-}} e^{+} \nu_{e}, \eta_{\eta \pi^{+} \pi^{-}}^{\prime} e^{+} \nu_{e}$, and $\eta_{\gamma \rho^{0}}^{\prime} e^{+} \nu_{e}$ (the same sequence later), respectively, by repeating fits with different fit ranges and different signal and background shapes. The ST efficiencies may be different due to the different multiplicities in the tag environments, leading to incomplete cancellation of the systematic uncertainties associated with the ST selection. The associated uncertainty is assigned as $0.4 \%, 0.3 \%, 0.3 \%, 0.3 \%$, from studies of the efficiency differences for tracking and PID of $K^{ \pm}$ and $\pi^{ \pm}$as well as the selection of neutral particles between data and MC simulation in different environments. The uncertainty due to the $M_{\eta^{\prime} e^{+}}$requirement is found to be negligible. The uncertainty due to peaking background is assigned to be $1.4 \%$ by varying its size by $\pm 1 \sigma$ of the corresponding BF. The uncertainties due to the quoted BFs, $0.9 \%, 1.4 \%, 1.8 \%$, and $1.9 \%$ of $\eta^{(\prime)}$ decays [22] are also considered. For each decay, the total systematic uncertainty is determined to be $2.7 \%, 3.3 \%, 3.4 \%$, and $4.0 \%$ by adding all these uncertainties in quadrature.
With the BFs measured in this work, we determine the $\mathrm{BF}$ ratio $\mathcal{R}_{\eta^{\prime} / \eta}^{D_{s}^{+}}=\mathcal{B}_{D_{s}^{+} \rightarrow \eta e^{+} \nu_{e}} / \mathcal{B}_{D_{s}^{+} \rightarrow \eta^{\prime} e^{+} \nu_{e}}=$ $0.355 \pm 0.033_{\text {stat }} \pm 0.015_{\text {syst }}$, where the systematic uncertainties on the ST yield and due to the photon from $D_{s}^{*+}$, FSR recovery, tracking and PID of $e^{+}$cancel. Using these BFs and $\mathcal{B}_{D^{+} \rightarrow \eta^{(\prime)} e^{+} \nu_{e}}$ reported in Ref. [28], we determine the $\eta-\eta^{\prime}$ mixing angle to be $\phi_{P}=$ $\left(40.1 \pm 2.1_{\text {stat }} \pm 0.7_{\text {syst }}\right)^{\circ}$. This result is consistent with previous measurements using $D \rightarrow \eta^{(\prime)} e^{+} \nu_{e}$ decays [9] and $\psi \rightarrow \gamma \eta^{(\prime)}$ decays [10] within uncertainties.

To study the $D_{s}^{+} \rightarrow \eta^{(\prime)} e^{+} \nu_{e}$ dynamics, the candidate events are divided into various $q^{2}$ intervals. The measured partial decay width $\Delta \Gamma_{\mathrm{msr}}^{i}$ in the $i$ th $q^{2}$ interval is determined by $\Delta \Gamma_{\mathrm{msr}}^{i} \equiv \int_{i}\left(d \Gamma / d q^{2}\right) d q^{2}=\left(N_{\mathrm{pro}}^{i} / \tau_{D_{s}^{+}} \times N_{\mathrm{ST}}^{\text {tot }}\right)$, where $\tau_{D_{s}^{+}}$is the lifetime of the $D_{s}^{+}$meson [22,29], and $N_{\text {pro }}^{i}$ is the DT yield produced in the $i$ th $q^{2}$ interval, calculated by $N_{\text {pro }}^{i}=\sum_{j}^{m}\left(\epsilon^{-1}\right)_{i j} N_{\mathrm{obs}}^{j}$. Here $m$ is the number of $q^{2}$ intervals, $N_{\mathrm{obs}}^{j}$ is the observed DT yield obtained from similar fits to the $\mathrm{MM}^{2}$ distribution as described previously, and $\epsilon_{i j}$ is the efficiency matrix determined from signal MC events and is given by $\epsilon_{i j}=\sum_{k}\left[\left(1 / N_{\mathrm{ST}}^{\mathrm{tot}}\right) \times\left(N_{\text {rec }}^{i j} / N_{\text {gen }}^{j}\right)_{k} \times\right.$ $\left.\left(N_{\mathrm{ST}}^{k} / \epsilon_{\mathrm{ST}}^{k}\right)\right]$, where $N_{\text {rec }}^{i j}$ is the DT yield generated in the $j$ th $q^{2}$ interval and reconstructed in the $i$ th $q^{2}$ interval, $N_{\text {gen }}^{j}$ is the total signal yield generated in the $j$ th $q^{2}$ interval, and $k$ sums over all tag modes. See Tables I and II of Ref. [30] for details about the range, $N_{\mathrm{obs}}^{i}, N_{\mathrm{prd}}^{i}$, and $\Delta \Gamma_{\mathrm{msr}}^{i}$ of each $q^{2}$ interval for $D_{s}^{+} \rightarrow \eta e^{+} \nu_{e}$ and $D_{s}^{+} \rightarrow \eta^{\prime} e^{+} \nu_{e}$, respectively.

In theory, the differential decay width can be expressed

$$
\frac{d \Gamma\left(D_{s}^{+} \rightarrow \eta^{(\prime)} e^{+} \nu\right)}{d q^{2}}=\frac{G_{F}^{2}\left|V_{\mathrm{cs}}\right|^{2}}{24 \pi^{3}}\left|f_{+}^{\eta^{(\prime)}}\left(q^{2}\right)\right|^{2}\left|p_{\eta^{(\prime)}}\right|^{3},
$$

where $\left|p_{\eta^{\prime \prime}}\right|$ is the magnitude of the meson 3-momentum in the $D_{s}^{+}$rest frame and $G_{F}$ is the Fermi constant. In the modified pole model [31],

$$
f_{+}\left(q^{2}\right)=\frac{f_{+}(0)}{\left(1-\frac{q^{2}}{M_{\text {pole }}^{2}}\right)\left(1-\alpha \frac{q^{2}}{M_{\text {pole }}^{2}}\right)},
$$

where $M_{\text {pole }}$ is fixed to $M_{D_{s}^{*+}}$ and $\alpha$ is a free parameter. Setting $\alpha=0$ and leaving $M_{\text {pole }}$ free, it is the simple

TABLE II. Results of the fits to $\Delta \Gamma_{\mathrm{msr}}^{i}$. Uncertainties on the least significant digits are shown in parentheses, where the first (second)

\begin{tabular}{|c|c|c|c|c|c|c|c|c|c|}
\hline \multirow[b]{2}{*}{ Case } & \multicolumn{3}{|c|}{ Simple pole } & \multicolumn{3}{|c|}{ Modified pole } & \multicolumn{3}{|c|}{ Series 2 Par. } \\
\hline & $f_{+}^{\eta_{+}^{(\prime)}(0)\left|V_{\mathrm{cs}}\right|}$ & $M_{\text {pole }}$ & $\overline{\chi^{2} / N_{\text {d.o.f. }} .}$ & $f_{+}^{\eta_{+}^{(\prime)}(0)\left|V_{\mathrm{cs}}\right|}$ & $\alpha$ & $\overline{\chi^{2} / N_{\text {d.o.f. }} .}$ & $\overline{f_{+}^{\eta^{(1)}}(0)\left|V_{\mathrm{cs}}\right|}$ & $r_{1}$ & $\chi^{2} / N_{\text {d.o.f }}$. \\
\hline$\overline{\eta e^{+} \nu_{e}}$ & $0.4505(45)(31)$ & $3.759(84)(45)$ & $12.2 / 14$ & $0.4457(46)(34)$ & $0.304(44)(22)$ & $11.4 / 14$ & $0.4465(51)(35)$ & $-2.25(23)(11)$ & $11.5 / 14$ \\
\hline$\eta^{\prime} e^{+} \nu_{e}$ & $0.483(42)(10)$ & $1.88(60)(08)$ & $1.8 / 4$ & $0.481(44)(10)$ & $1.62(91)(13)$ & $1.8 / 4$ & $0.477(49)(11)$ & $-13.1(76)(10)$ & $1.9 / 4$ \\
\hline
\end{tabular}
uncertainties are statistical (systematic). $N_{\text {d.o.f }}$ is the number of degrees of freedom. 
TABLE III. Comparison of the measured $f_{+}^{\eta^{(\prime)}}(0)$ with the theoretical calculations. Errors on the least significant digits are shown in parentheses. For the LQCD model, the errors are statistical only, while ${ }^{A(B)}$ assume $M_{\pi}=470(370) \mathrm{MeV}$.

\begin{tabular}{ccccccccccc}
\hline \hline & CLFQM [44] & CQM [45] & CCQM [46] & 3PSR [47] & LCSR [48] & LCSR [7] & LQCD $^{A}$ [6] & LQCD $^{B}$ [6] & LCSR [8] & BESIII \\
\hline$f_{+}^{\prime}(0)$ & 0.76 & 0.78 & $0.78(12)$ & $0.50(4)$ & $0.45(15)$ & $0.432(33)$ & $0.564(11)$ & $0.542(13)$ & $0.495(30)$ & $0.4576(70)$ \\
$f_{+}^{\eta^{\prime}}(0)$ & $\cdots$ & 0.78 & $0.73(11)$ & $\ldots$ & $0.55(18)$ & $0.520(88)$ & $0.437(18)$ & $0.404(25)$ & $0.558(47)$ & $0.490(51)$ \\
\hline \hline
\end{tabular}

pole model [32]. In the two-parameter (2 Par.) series expansion [31]

$$
f_{+}\left(q^{2}\right)=\frac{1}{A\left(q^{2}\right)} \frac{f_{+}(0) A(0)}{1+B(0)}\left[1+B\left(q^{2}\right)\right] .
$$

Here, $\quad A\left(q^{2}\right)=P\left(q^{2}\right) \Phi\left(q^{2}, t_{0}\right), \quad B\left(q^{2}\right)=r_{1}\left(t_{0}\right) z\left(q^{2}, t_{0}\right)$, $t_{0}=t_{+}\left(1-\sqrt{1-t_{-} / t_{+}}\right), t_{ \pm}=\left(M_{D_{s}^{+}} \pm M_{\eta}\right)$, and $r_{k}$ is a free parameter. The functions $P\left(q^{2}\right), \Phi\left(q^{2}, t_{0}\right)$, and $z\left(q^{2}, t_{0}\right)$ are defined following Ref. [31].

For each SL decay, the product $f_{+}(0)\left|V_{\text {cs }}\right|$ and one other parameter, $M_{\text {pole }}, \alpha$, or $r_{1}$, are determined by constructing and minimizing

$$
\chi^{2}=\sum_{i j=1}^{m}\left(\Delta \Gamma_{\mathrm{msr}}^{i}-\Delta \Gamma_{\exp }^{i}\right) C_{i j}^{-1}\left(\Delta \Gamma_{\mathrm{msr}}^{j}-\Delta \Gamma_{\exp }^{j}\right),
$$

with $\Delta \Gamma_{\mathrm{msr}}^{i}$ and the theoretically expected value $\Delta \Gamma_{\exp }^{i}$, where $C_{i j}=C_{i j}^{\mathrm{stat}}+C_{i j}^{\mathrm{syst}}$ is the covariance matrix of $\Delta \Gamma_{\mathrm{msr}}^{i}$ among $q^{2}$ intervals, as shown in Tables III and IV in Ref. [30]. For each $\eta^{(/)}$subdecay, the statistical covariance matrix is constructed with the statistical uncertainty in each $q^{2}$ interval $\left[\sigma\left(N_{\mathrm{obs}}^{\alpha}\right)\right]$ as $C_{i j}^{\text {stat }}=$ $\left(1 / \tau_{D_{s}^{+}} N_{\mathrm{ST}}^{\mathrm{tot}}\right)^{2} \sum_{\alpha} \epsilon_{i \alpha}^{-1} \epsilon_{j \alpha}^{-1}\left[\sigma\left(N_{\mathrm{obs}}^{\alpha}\right)\right]^{2}$. The systematic covariance matrix is obtained by summing all the covariance matrices for all systematic uncertainties, which are all constructed with the systematic uncertainty in each $q^{2}$ interval $\left[\delta\left(\Delta \Gamma_{\mathrm{msr}}^{i}\right)\right]$ as $C_{i j}^{\text {syst }}=\delta\left(\Delta \Gamma_{\mathrm{msr}}^{i}\right) \delta\left(\Delta \Gamma_{\mathrm{msr}}^{j}\right)$. Here, an additional systematic uncertainty in $\tau_{D_{s}^{+}}(0.8 \%)$ [22,29] is involved besides those in the $\mathrm{BF}$ measurements.

The $\Delta \Gamma_{\mathrm{msr}}^{i}$ measured by the two $\eta^{(\prime)}$ subdecays are fitted simultaneously, with results shown in Fig 3. In the fits, the $\Delta \Gamma_{\mathrm{msr}}^{i}$ becomes a vector of length $2 \mathrm{~m}$. Uncorrelated systematic uncertainties are from tag bias, MC statistics, quoted BFs, $\eta$ (and $\pi^{0}$ ) reconstruction, and FF parametrization, while other systematic uncertainties are fully correlated. Table II summarizes the fit results, where the obtained $f_{+}^{\eta^{(\prime)}}(0)\left|V_{\text {cs }}\right|$ with different FF parametrizations are consistent with each other.

Combining $\left|V_{\mathrm{cs}}\right|=0.97343 \pm 0.00015$ from the global fit in the SM [22] with $f_{+}^{\eta^{(\prime)}}(0)\left|V_{\text {cs }}\right|$ extracted from the two-parameter series expansion, we determine $f_{+}^{\eta}(0)=$ $0.4576 \pm 0.0054_{\text {stat }} \pm 0.0045_{\text {syst }}$ and $f_{+}^{\eta^{\prime}}(0)=0.490 \pm$ $0.050_{\text {stat }} \pm 0.011_{\text {syst }}$. Table III compares the measured
FFs with various theoretical calculations within uncertainties. When combining $f_{+}^{\eta}(0)$ and $f_{+}^{\eta^{\prime}}(0)$ calculated from Ref. [7], we obtain $\left|V_{\text {cs }}\right|=1.031 \pm 0.012_{\text {stat }} \pm 0.009_{\text {syst }} \pm$ $0.079_{\text {theo }}$ and $0.917 \pm 0.094_{\text {stat }} \pm 0.021_{\text {syst }} \pm 0.155_{\text {theo }}$, respectively. These results agree with the measurements of $\left|V_{\text {cs }}\right|$ using $D \rightarrow \bar{K} \ell^{+} \nu_{\ell}$ [33-38] and $D_{s}^{+} \rightarrow \ell^{+} \nu_{\ell}$ decays [39-43] within uncertainties.

In summary, by analyzing a data sample of $3.19 \mathrm{fb}^{-1}$ taken at $E_{\text {c.m. }}=4.178 \mathrm{GeV}$ with the BESIII detector, we measure the absolute BFs of $D_{s}^{+} \rightarrow \eta^{(\prime)} e^{+} \nu_{e}$ with a DT method. The precision is improved by a factor of 2 compared to the world average values. Using these BFs and $\mathcal{B}\left(D^{+} \rightarrow \eta^{(\prime)} e^{+} \nu_{e}\right)$ measured in our previous work [28], we determine the $\eta-\eta^{\prime}$ mixing angle $\phi_{P}$, which provides complementary data to constrain the gluon component in the $\eta^{\prime}$ meson. From an analysis of the dynamics in $D_{s}^{+} \rightarrow \eta^{(\prime)} e^{+} \nu_{e}$, the products of $f_{+}^{\eta^{(\prime)}}(0)\left|V_{\mathrm{cs}}\right|$ are determined for the first time. Furthermore, by taking $\left|V_{\text {cs }}\right|$ from a standard model fit (CKMFITTER [22]) as input, we determine the FF at zero momentum transfer $f_{+}^{\eta^{(\prime)}}(0)$ for the first time. The obtained FFs provide important data to distinguish various theoretical calculations [6-8,45-48]. Alternatively, we also determine $\left|V_{\text {cs }}\right|$ with $D_{s}^{+} \rightarrow$ $\eta^{(\prime)} e^{+} \nu_{e}$ decays for the first time, by taking values for $f_{+}^{\eta^{(1)}}(0)$ calculated in theory. Our result on $\left|V_{\mathrm{cs}}\right|$ together with those measured by $D \rightarrow \bar{K} \ell^{+} \nu_{\ell}$ and $D_{s}^{+} \rightarrow \ell^{+} \nu_{\ell}$ are important to test the unitarity of the CKM matrix.

The BESIII Collaboration thanks the staff of BEPCII and the IHEP computing center for their strong support. This work is supported in part by National Key Basic Research Program of China under Contract No. 2015CB856700; National Natural Science Foundation of China (NSFC) under Contracts No. 11335008, No. 11425524, No. 11625523, No. 11635010, and No. 11735014; the Chinese Academy of Sciences (CAS) Large-Scale Scientific Facility Program; the CAS Center for Excellence in Particle Physics (CCEPP); Joint LargeScale Scientific Facility Funds of the NSFC and CAS under Contracts No. U1632109, No. U1532257, No. U1532258, and No. U1732263; CAS Key Research Program of Frontier Sciences under Contracts No. QYZDJSSW-SLH003 and No. QYZDJ-SSW-SLH040; 100 Talents Program of CAS; INPAC and Shanghai Key Laboratory for Particle Physics and Cosmology; German Research 
Foundation DFG under Contracts No. Collaborative Research Center CRC 1044 and No. FOR 2359; Istituto Nazionale di Fisica Nucleare, Italy; Koninklijke Nederlandse Akademie van Wetenschappen (KNAW) under Contract No. 530-4CDP03; Ministry of Development of Turkey under Contract No. DPT2006K120470; National Science and Technology fund; The Swedish Research Council; U.S. Department of Energy under Contracts No. DE-FG02-05ER41374, No. DE-SC0010118, No. DE-SC-0010504, and No. DE-SC-0012069; University of Groningen (RuG) and the Helmholtzzentrum fuer Schwerionenforschung GmbH (GSI), Darmstadt.

${ }^{a} A l s o$ at Bogazici University, 34342 Istanbul, Turkey.

${ }^{\mathrm{b}}$ Also at the Moscow Institute of Physics and Technology, Moscow 141700, Russia.

${ }^{c}$ Also at the Functional Electronics Laboratory, Tomsk State University, Tomsk 634050, Russia.

${ }^{\mathrm{d}}$ Also at the Novosibirsk State University, Novosibirsk 630090, Russia.

"Also at the NRC "Kurchatov Institute", PNPI, Gatchina 188300, Russia.

${ }^{\mathrm{f}}$ Also at Istanbul Arel University, 34295 Istanbul, Turkey.

${ }^{\mathrm{g}}$ Also at Goethe University Frankfurt, 60323 Frankfurt am Main, Germany.

${ }^{\mathrm{h}}$ Also at Key Laboratory for Particle Physics, Astrophysics and Cosmology, Ministry of Education; Shanghai Key Laboratory for Particle Physics and Cosmology; Institute of Nuclear and Particle Physics, Shanghai 200240, People's Republic of China.

${ }^{i}$ Also at Government College Women University, Sialkot51310, Punjab, Pakistan.

${ }^{j}$ Also at Key Laboratory of Nuclear Physics and Ion-beam Application (MOE) and Institute of Modern Physics, Fudan University, Shanghai 200443, People's Republic of China. ${ }^{\mathrm{k}}$ Also at Harvard University, Department of Physics, Cambridge, MA, 02138, USA.

[1] L. Riggio, G. Salerno, and S. Simula, Eur. Phys. J. C 78, 501 (2018).

[2] J. Zhang, C. X. Yue, and C. H. Li, Eur. Phys. J. C 78, 695 (2018).

[3] Y. Fang, G. Rong, H. L. Ma, and J. Y. Zhao, Eur. Phys. J. C 75, 10 (2015)

[4] N. H. Christ, C. Dawson, T. Izubuchi, C. Jung, Q. Liu, R. D. Mawhinney, C. T. Sachrajda, A. Soni, and R. Zhou, Phys. Rev. Lett. 105, 241601 (2010).

[5] J. J. Dudek, R. G. Edwards, B. Joo, M. J. Peardon, D. G. Richards, and C.E. Thomas, Phys. Rev. D 83, 111502 (2011).

[6] G. S. Bali, S. Collins, S. Dürr, and I. Kanamori, Phys. Rev. D 91, 014503 (2015).

[7] N. Offen, F. A. Porkert, and A. Schäfer, Phys. Rev. D 88, 034023 (2013).

[8] G. Duplančić and B. Melic, J. High Energy Phys. 11 (2015) 138.

[9] C. Di Donato, G. Ricciardi, and I. I. Bigi, Phys. Rev. D 85, 013016 (2012).
[10] F. Ambrosino et al. (KLOE Collaboration), Phys. Lett. B 648, 267 (2007).

[11] R. Aaij et al. (LHCb Collaboration), J. High Energy Phys. 01 (2015) 024.

[12] G. Brandenburg et al. (CLEO Collaboration), Phys. Rev. Lett. 75, 3804 (1995).

[13] J. Yelton et al. (CLEO Collaboration), Phys. Rev. D 80, 052007 (2009).

[14] J. Hietala, D. Cronin-Hennessy, T. Pedlar, and I. Shipsey, Phys. Rev. D 92, 012009 (2015).

[15] M. Ablikim et al. (BESIII Collaboration), Phys. Rev. D 94, 112003 (2016).

[16] Charge conjugated modes are implied throughout this Letter.

[17] M. Ablikim et al. (BESIII Collaboration), Nucl. Instrum. Methods Phys. Res., Sect. A 614, 345 (2010).

[18] X. Li et al., Radiat. Detect. Technol. Methods. 1, 13 (2017).

[19] Y. X. Guo et al., Radiat. Detect. Technol. Methods. 1, 15 (2017).

[20] S. Agostinelli et al. (GEANT4 Collaboration), Nucl. Instrum. Methods Phys. Res., Sect. A 506, 250 (2003).

[21] R. G. Ping, Chin. Phys. C 38, 083001 (2014).

[22] M. Tanabashi et al. (Particle Data Group), Phys. Rev. D 98, 030001 (2018).

[23] D. J. Lange, Nucl. Instrum. Methods Phys. Res., Sect. A 462, 152 (2001); R. G. Ping, Chin. Phys. C 32, 599 (2008).

[24] J. C. Chen, G. S. Huang, X. R. Qi, D. H. Zhang, and Y. S. Zhu, Phys. Rev. D 62, 034003 (2000).

[25] O. G. Tchikilev, Phys. Lett. B 471, 400 (2000); 478, 459(E) (2000).

[26] M. Ablikim et al. (BESIII Collaboration), Phys. Rev. Lett. 122, 071802 (2019).

[27] M. Ablikim et al. (BESIII Collaboration), Phys. Rev. D 83, 112005 (2011).

[28] M. Ablikim et al. (BESIII Collaboration), Phys. Rev. D 97, 092009 (2018).

[29] R. Aaij et al. (LHCb Collaboration), Phys. Rev. Lett. 119, 101801 (2017).

[30] See Supplement Material at http://link.aps.org/ supplemental/10.1103/PhysRevLett.122.121801 for measured partial width, statistical and systematic covariance matrices.

[31] T. Becher and R. J. Hill, Phys. Lett. B 633, 61 (2006).

[32] D. Becirevic and A. B. Kaidalov, Phys. Lett. B 478, 417 (2000).

[33] M. Ablikim et al. (BESIII Collaboration), Phys. Rev. D 92, 072012 (2015)

[34] M. Ablikim et al. (BESIII Collaboration), Phys. Rev. D 92, 112008 (2015).

[35] M. Ablikim et al. (BESIII Collaboration), Phys. Rev. Lett. 122, 011804 (2019).

[36] D. Besson et al. (CLEO Collaboration), Phys. Rev. D 80, 032005 (2009).

[37] B. Aubert et al. (BABAR Collaboration), Phys. Rev. D 76, 052005 (2007).

[38] L. Widhalm et al. (Belle Collaboration), Phys. Rev. Lett. 97, 061804 (2006).

[39] M. Ablikim et al. (BESIII Collaboration), Phys. Rev. D 94, 072004 (2016). 
[40] A. Zupanc et al. (Belle Collaboration), J. High Energy Phys. 09 (2013) 139.

[41] P. del Amo Sanchez et al. (BABAR Collaboration), Phys. Rev. D 82, 091103 (2010).

[42] P. U. E. Onyisi et al. (CLEO Collaboration), Phys. Rev. D 79, 052002 (2009).

[43] P. Naik et al. (CLEO Collaboration), Phys. Rev. D 80, 112004 (2009).
[44] R. C. Verma, J. Phys. G 39, 025005 (2012).

[45] D. Melikhov and B. Stech, Phys. Rev. D 62, 014006 (2000).

[46] N. R. Soni, M. A. Ivanov, J. G. Körner, J. N. Pandya, P. Santorelli, and C. T. Tran, Phys. Rev. D 98, 114031 (2018).

[47] P. Colangelo and F. De Fazio, Phys. Lett. B 520, 78 (2001).

[48] K. Azizi, R. Khosravi, and F. Falahati, J. Phys. G 38, 095001 (2011). 